\title{
Pain management \& opioid dose reduction with topical sevoflurane instillations in intractable venous ulcers: a case report
}

\author{
F. Dámaso Fernández-Ginés ${ }^{\circledR 1 *}$, Manuel Cortiñas-Saenz ${ }^{2}$, \\ Desirée Agudo-Ponce², José Antonio Morales-Molina ${ }^{3}$, \\ Carmen Fernández Sánchez ${ }^{1}$, Francisco Sierra-Garcia ${ }^{1}$ \\ ${ }^{1}$ Pharmacy Department, Torrecárdenas University Hospital, Almería, Spain, \\ ${ }^{2}$ Anesthesiology and Pain Management Department, Torrecárdenas University Hospital, \\ Almeria, ${ }^{3}$ Pharmacy Department, Public Health Agency of Poniente, El ejido,
}

\begin{abstract}
Vascular ulcers (VU) constitute a major cause of pain and disability, and significantly compromise quality of life. VU have a natural tendency to become chronic and in many cases exhibit anunsatisfactoryresponse to many of the standard therapeutic options.The case of a 73 year-old Caucasian female with severe pain and poorly-controlled pain (Visual Analogic Scale-VAS- of 8-9) due to three lower leg long-standing VUs is reported and discussed herein. The patient was treated with topical instillations of undiluted sevoflurane as per institutional off-label protocol (starting doses of $1 \mathrm{~mL} / \mathrm{cm}^{2}$ twice a day, and up-titrated according to response to a maximum of $7 \mathrm{~mL}$ twice daily). The VAS score dropped to 0-1 shortly after initiation of therapy and remained stable throughout treatment up until the closure of the observations. Subsequently, opioid therapy was gradually tapered down and ultimately abandoned.Sevoflurane application resulted on adequate and sustained pain management of refractory VU, with no significant side effects. On account of its beneficial effectivity and safety profiles, topical sevoflurane emerges as an add-on alternative for the long-term management of VU, and potentially other painful conditions.
\end{abstract}

Keywords: Pain,vascular ulcer. Topical drug administration. Opioids. Sevoflurane.

\section{INTRODUCTION}

Vascular ulcers (VU) are chronic skin lesions involving loss of tissue integrity induced by an underlying pathologic process of vascular origin. VU generally have little tendency to heal spontaneously, and can be debilitating and hinder mobility (Fitzpatrik, Bernhard, Cropley, 1999).

Although figures vary substantially according to the source, it is estimated that VU will affect between 1 and $2 \%$ of all individuals during their lifetime (Järbrink et al,

*Correspondence: F. D. Fernández-Ginés. Pharmacy Department. Torrecárdenas University Hospital. Hermanos Donantes de Sangre S/N. 04009- Almería, Spain
1999). VU represent a serious healthcare problem with deep socio-economic consequences (Herber, Schnepp, Rieger, 2007; Andrea, 2016; Vowden, Vowden, 2016).

The therapeutic management of VU is usually multi-disciplinary and includes basic wound hygiene, compression therapy, use of dressings, debridement surgery, antibiotic treatment, pentoxifylline to improve microcirculatory blood flow, and pain control (Andrea, 2016).

Pain management is complex and often requires the use of high-dose opioids, which represent the mainstay of therapy, usually in combination with other oral or topical agents, albeit the role of the latter remains unclear (Rüger et al, 2008; Briggs, Nelson, Martyn 2012). Recently, several studies have reported the use of topical 
instillations of the inhaled anaesthetic sevoflurane in the management of VU-associated pain (Gerónimo, Martínez, Martínez, 2011; Martínez, Gerónimo, 2011; Chu et al, 2008; Fernández et al, 2017; Fernández et al, 2018). The case of a female patient treated with topical sevoflurane in the management of refractory VU is reported and discussed herein.

\section{MATERIAL AND METHODS}

A 73 year-old Caucasian female was referred to the Pain Unit complaining of severe pain associated to three long-standing VU ( $>24$ months), located in the right lowerlegand measuring between 3 and $7 \mathrm{~cm}^{2}$. The baseline Visual Analogic Scale (VAS) was consistently reported as 8-9 despite regular painkillers (see medications below). Pain had both somatic and neuropathic features and was exacerbated by mobilisation and daily toileting of wounds, thus preventing daily activities and hampering night-time rest. Her performance status (PS) at presentation -a scale used to assess quality of life and patient's ability to perform routine tasks- was 2; ranges from 0 to 4 , with 0 being fully functional and asymptomatic, and 4 being bedridden. No itching, oozing, or other signs of over-infection were observable on examination (wound swab culture showed no bacterial growth after seven days). The past medical history was also significant for hypertension, type 2 diabetes, dyslipidemia, non-revascularizable acute coronary syndrome, gastric ulcer, chronic kidney disease, and Fontaine grade IV vascular peripheral disease.

The patient had had previous poor tolerance to morphine and oxycodone (dizziness, vomit, constipation, and drowsiness), which was non responsive to opioid rotation. Regular analgesia at time of presentation included: paracetamol 4g/day, metamizole 460-575g/ day, and transdermal buprenorphine $52 \mu \mathrm{g} / \mathrm{h}$ (1 patch every 4 days).

Consent to receive treatment with off-label sevoflurane (Sevorane ${ }^{\circledR}$, Abbvie, Campo verde d'Aprilia, Italy) instillations as per institutional protocol was obtained and documented in the medical notes. After wound cleaning with sterile saline, the patient received twice daily topical undiluted sevoflurane applications directly on the sore tissue. Applications were carried out with patient lying face-down, allowing the formulation to completely cover the injured areas, and using a sterile gauze to prevent contact from the overflowing fluid with surrounding healthy skin. Doses were adjusted subjected to response, achieving a maximum of $7 \mathrm{~mL}$ per procedure.

A marked and durable $(\sim 12 \mathrm{~h})$ reduction in pain scores was observed immediately after each use (see Figure 1),enabling debridement and general wound toileting. Treatment continued in an ambulatory setting for a total of 111 days. A reduction in the extent of the lesions using the Kundin scale and the Gilman's equation was noted (figure 1) (Kundin, 1985; Gilman, 1990). Average PS shortly after initiation of therapy was $0-1$. Opioid therapy was gradually tapered downand eventually discontinued, with only paracetamol as required for breakthrough pain. No other changes to regular medications were required. Due to the risk of systemic absorption of sevoflurane, routine blood tests including renal and liver profiles was requested throughout treatment, as well as wound swabs. All tests remained unremarkable (Figure 2).

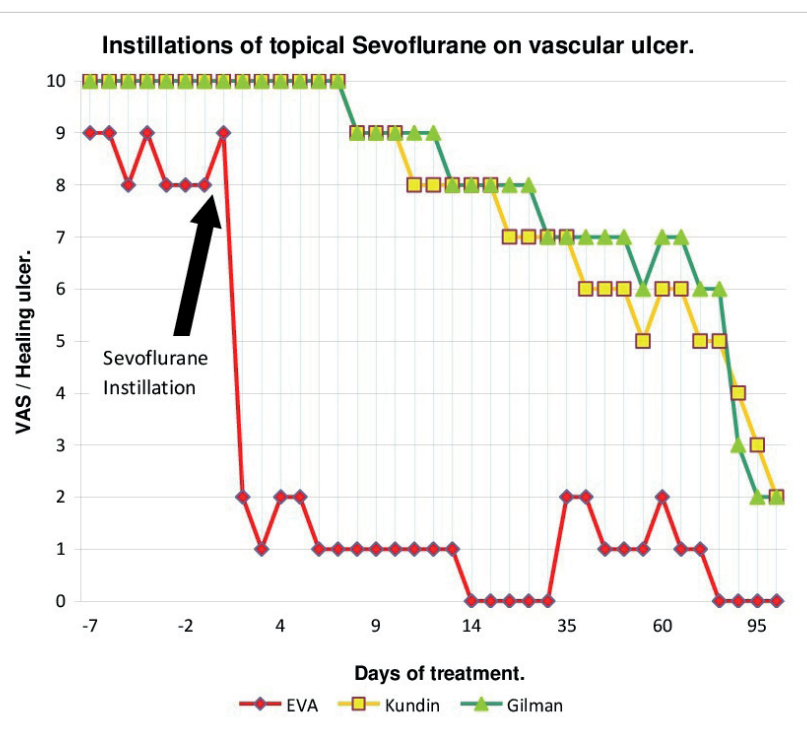

FIGURE 1 - Pain evolution using a Visual Analogue Scale (VAS) and percentage of healing of the vascular ulcer using the Kundin equation (Ulcer surface $=$ Long $\diamond$ Width $\diamond 0.875$ ) and Gilman (linear healing ratio $=$ ulcer area $/$ Perimeter ulcer). 


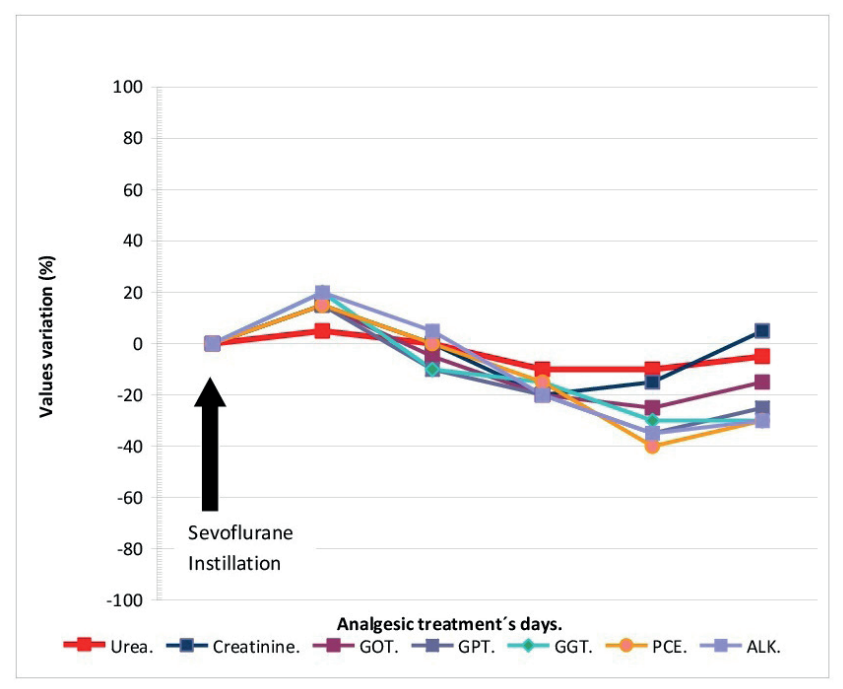

FIGURE 2 - Variation during treatment with instillation of topical sevoflurane renal function (Urea and Creatinine), liver damage by Glutamic Pyruvic Transaminase (GPT) and Glutamic Oxaloacetic Transaminase(GOT), Gammaglutamyltranspeptidase (GGT), Plasma cholinesterase (PCE) as evaluation of the liver reserve and Alkaline Phosphatase (ALK) to evidence cholestasis.

\section{RESULTS AND DISCUSSION}

In the case reported here, a patient with longstanding intractable VU-related pain experienced a marked and durable response to direct topical sevoflurane applications. This was evidenced by a significant reduction in pain scores immediately after each intervention, which lasted approximately 12 hours. Moreover, wound size measures showed a steady decrease, which continued at the point of closure of the observations, as detailed in figure 1. Not surprisingly, this was accompanied by a noticeable improvement in the patient's PS and quality of life, though no formal assessment of the latter was conducted.

These results are consistent with previous reports on the use of topical sevoflurane in varicose and ischaemic ulcers (Gerónimo, Martínez, Martínez, 2011; Martínez, Gerónimo, 2011; Chu et al, 2008; Fernández et al, 2017; Fernández et al, 2018).In the same line though conducted in a somewhat different setting, other authors have reported similar effects after subcutaneous sevoflurane injections (Fernández et al, 2018).

From a safety perspective, none of the side effects reported in previous studies with topical sevoflurane (namely mild-to-moderate reddening and itching of surrounding skin) were observed in this patient. These could be due to a careful application technique.Regular clinical examinations and analytical findings were equally unremarkable.

Another advantage of topical sevoflurane was the gradual and sustained reduction of opioid doses, with no reported episodes of related toxicity during therapy, which arguably represent one of the main disadvantages of their long-term use. Furthermore, the relatively easiness of application and treatment acceptance facilitated its transition to primary care or domiciliary use, provided patients and district nurses receive adequate training.

In this case, sevoflurane enabled effective control of superficial and deep nociceptive and neuropathic pain, as well as wound toileting and debridement-induced breakthrough pain. The mechanism whereby sevoflurane (alongside other halogenated anaesthetics) seems to exert such central and peripheral analgesic action is not completely understood though. Some authors postulate that sevoflurane induces muscle relaxation and reduces pain sensitivity by decreasing the extent of gap junctionmediated cell-cell coupling and altering the activity of the channels that underlie the action potential(Argoff, 2013). In addition, sevoflurane possesses vasodilatory properties which could help counteract the existing hypertension (main process underpinning the development of VU). More importantly, the increase in permeability could in turn stimulate tropism of blood cells and release of chemotactic factors necessary for wound healing (Preckel, 2005).In addition, sevoflurane exhibits in vitro and in vivo broad-spectrum bactericidal action, which might equally contribute to the proepithelising process by preventing overgrowth of skin pathogens (Martínez, Gerónimo, Martínez, 2017; Imbernón et al, 2017). This was supported by the absence of bacterial colonies in wound swab cultures obtained regularly throughout the treatment. Finally, treatment compliance (during domiciliary administrations) was acceptable, despite twice daily applications. In our case, after 111 days of treatment, no systemic or local adverse effects were observed.

Overall, these findings are in line with emerging evidence suggesting that sevoflurane may possess analgesic and healing actions through mechanisms not yet fully elucidated. Subsequently, sevoflurane could play a role in the management of complicated VU. In this regard, further studies aimed at assessing its longterm efficacy and safety, as well as its impact in quality 
of life in a larger cohort are under development and should help clarify some of these uncertainties.

\section{REFERENCES}

Nelson EA, Adderley, U. Venous leg ulcers. BMJ Clin Evid. 2016;2016:1902.

Argoff CE. Topical analgesics in the management of acute and chronic pain. MayoClinProc. 2013;88(2):195-205.

Briggs M, Nelson EA, Martyn-St James M. Topical agents or dressings for pain in venous leg ulcers. Cochrane database of systematic reviews.2012;11.CD001177

Chu CC, Wu SZ, Su WL, Shieh JP, kao CH, Ho ST, Wang JJ. Subcutaneous injection of inhaled anesthetics produces cutaneous analgesia. Can J Anaesth. 2008; 55(5):290-294.

Fernández-Ginés FD, Cortiñas-Sáenz M, Mateo-Carrasco H, de Aranda AN, Navarro-Muñoz E, et al. Efficacy and safety of topical sevoflurane in the treatment of chronic skin ulcers. Am J Eur J Hosp Pharm. 2018; 26(4):229-232.

Fernández-Ginés FD, Cortiñas-Sáenz M, Navajas-Gómez de Aranda A,Maria del Carmen Navas-Martinez4, José Antonio Morales-Molina, et al.Palliative analgesia with topical sevoflurane in cancer-related skin ulcers: a case report. Eur J Hosp Pharm.2018;0:1-4.

Fitzpatrik TB, Bernhard JD, Cropley TG. The structure of skin lesions and fundamentals of diagnosis. Fitzpatrik's dermatology in general medicine. New York: McGraw-Hill 1999. p.13-41.

Gerónimo M, Martínez A, Martínez M. Analgesic effect of topical sevoflurane on venous ulcer intractable pain. Phlebologie. 2011;40(2):95-97.

Gilman T. Parameter for measurement of wound closure. Wounds. 1990;2:95-101
JärbrinkK, Ni G, Sönnergren H, Schmidtchen A, Pang C, Bajpai R, Carl J. Prevalence and incidence of chronic wounds and related complications: a protocol for a systematic review. Syst Rev. 2016;5(1):152.

Kundin JL. Designing and developing a new measuring instrument. PerioperNurs Q. 1985;8(4):37-42.

Herber OR, Schnepp W, Rieger MA. A systematic review on the impact of leg ulceration on patient's quality of life. Health Qual Life Outcomes.2007;5(1):44

Imbernón-Moya A, Ortiz-de Frutos FJ, Sanjuan-Alvarez M, Portero-Sanchez I, Merinero-Palomares R, Alcazar V. Topical sevoflurane for chronic venous ulcers infected by multi-drugresistant organisms. Int Wound J. 2017;14(6):1388-1390.

Martínez-Serrano M, Gerónimo-Pardo M, MartínezMonsalve A, Crespo-Sánchez MD. Antibacterial effect of sevoflurane and isoflurane. RevEspQuimioter. 2017;30(2):8489.

Martínez A, Gerónimo M. Sevoflurano como anestésico local en herida isquémica de paciente cardiópata con insuficiencia respiratoria secundaria a morfina. Heridas y cicatrización. 2011;6:46-9.

Preckel B, Bolten J. Pharmacology of modern volatile anaesthetics. BestPract Res Clin Anaesthesiol. 2005;19(3):33148.

Rüger LJ, Irnich D, Abahji TN, Crispin A, Hoffman U, Lang PM. Characteristics of pain in patients with peripheral arterial disease. Pain. 2008;139(1):201-208.

Vowden P, Vowden K. The economic impact of hard-to-heal wounds: promoting practice change to address passivity in wound management. Wound Int, 2016;7(2):10-15

Received for publication on $15^{\text {th }}$ November 2018 Accepted for publication on $01^{\text {st }}$ February 2019 